\title{
Reliability sensitivity analysis with Monte Carlo methods
}

\author{
I. Papaioannou, K. Breitung \& D. Straub \\ Engineering Risk Analysis Group \\ Technische Universität München, Munich, Germany
}

\begin{abstract}
In many practical applications of structural reliability analysis, one is interested in knowing the sensitivity of the probability of failure to design parameters that enter the definition of the limit-state function. This information is required for example in reliability-based design optimization. Parameter sensitivities are obtained by FORM/SORM, in terms of the sensitivity of the respective probability approximation. However, in several cases FORM/SORM approximations are inaccurate or difficult to obtain. Hence, it is useful to obtain parameter sensitivities as a by-product of Monte Carlo methods. The derivative of the failure probability with respect to a parameter of the limit state function is given by a surface integral over the limit state surface. Application of Monte Carlo methods for the estimation of surface integrals is not straightforward. The surface integral can be computed applying sampling techniques that detect the failure surface, such as directional or line sampling. In this paper, we derive an approximation of the surface integral in terms of a domain integral. The domain integral can then be estimated through standard Monte Carlo or importance sampling simulation. A numerical example demonstrates the effectiveness of the proposed approximation for estimation with an importance sampling method.
\end{abstract}

\section{INTRODUCTION}

Structural reliability analysis requires the evaluation of the probability of failure, defined by the following $n$-fold integral:

$P_{f}=\int_{g(\mathbf{x}) \leq 0} f_{\mathbf{X}}(\mathbf{x}) d \mathbf{x}$

where $\mathbf{X}$ is an $n$-dimensional vector of random variables described by the joint PDF $f_{\mathbf{X}}(\mathbf{x})$ and $g(\mathbf{x}) \leq 0$ defines the failure event. The function $g(\mathbf{x})$ is usually termed limit-state function and it can include one or several distinct failure modes.

It is common to transform the random variables $\mathbf{X}$ to a probability space $\mathbf{U}$ consisting of independent standard normal random variables. This is achieved by an one-to-one transformation $\mathbf{U}=\mathbf{T}(\mathbf{X})$ (Hohenbichler \& Rackwitz 1981, Der Kiureghian \& Liu 1986). The probability of failure can be expressed in the transformed space as

$P_{f}=\int_{G(\mathbf{u}) \leq 0} \varphi_{n}(\mathbf{u}) d \mathbf{u}$

where $\varphi_{n}$ is the $n$-variate standard normal PDF and $G(\mathbf{u})=g\left(\mathbf{T}^{-1}(\mathbf{u})\right)$ is the limit-state function in the U-space.
The integral in Equation (1) can be evaluated by a variety of existing approaches (Ditlevsen \& Madsen 1996, Lemaire 2009). These include approximation methods such as the first/second order reliability methods (FORM/SORM), as well as simulation techniques based on the Monte Carlo method.

FORM/SORM approaches have been successfully applied to the solution of a large number of structural reliability problems. These methods are based on first/second order Taylor series approximations of the boundary of the limit-state function in the $\mathbf{U}$ space, performed at the point of the failure region with largest probability density. The evaluation of this so-called most probable failure point requires the solution of an optimization problem. The solution of this problem might become difficult in cases where the limit-state function is expressed in terms of a numerical model or when the dimension $n$ of the random variable space becomes large. Moreover, FORM/SORM solutions might become inaccurate in such high dimensional problems or in problems with highly nonlinear limit-state functions (Rackwitz 2001, Valdebenito et al. 2010).

On the other hand, the Monte Carlo method is a simple and robust technique, that is able to handle any limit-state function, independent of its complexity. Also, the efficiency of the Monte Carlo method in its standard form does not depend on the dimension 
of the random variable space. The classical Monte Carlo method estimates $P_{f}$ by generating samples of $\mathbf{X}$ and taking the sample mean of the indicator function $I(\mathbf{X})$, where $I(\mathbf{x})=1$ if $g(\mathbf{x}) \leq 0$ and $I(\mathbf{x})=0$ otherwise. The disadvantage of the crude Monte Carlo method is that its computational demands for assessing small probabilities are high - the number of samples required is inversely proportional to the probability of failure. However, this can be circumvented through application of variance reduction techniques.

In many practical applications of structural reliability, one is interested in knowing the effect of a change in a deterministic variable that enters the definition of the reliability problem on the computed probability of failure. This variable can either be a parameter of the distribution of the random variables or a parameter of the limit-state function. Sensitivities of the probability of failure in terms of both distribution and limit-state parameters are obtained as a byproduct of FORM/SORM solutions (Ditlevsen \& Madsen 1996, Breitung 1994). They involve the evaluation of firstor second-order derivatives of the transformation $\mathbf{T}($. and the limit-state function $g($.$) to the respective pa-$ rameters at the most probable failure point.

In this paper, we discuss the estimation of sensitivities of the probability of failure with Monte Carlo methods. We focus on sensitivities to limit-state parameters, such as deterministic design parameters, that are required for example in reliability-based design optimization with gradient methods. Application of Monte Carlo methods for the estimation of sensitivities to distribution parameters is a straightforward task, as will be discussed later on. In the following, we introduce the reliability sensitivity problem and review existing simulation approaches. We then discuss the application of existing variance reduction methods to reliability sensitivity estimation and introduce a novel approach for obtaining approximate reliability sensitivities with standard Monte Carlo methods.

\section{RELIABILITY SENSITIVITY ANALYSIS}

Let us consider two distinct sets of parameters, namely $\boldsymbol{\theta}_{f}$ and $\boldsymbol{\theta}_{g}$. The vector $\boldsymbol{\theta}_{f}$ contains parameters of the joint PDF of $\mathbf{X}$ and the vector $\boldsymbol{\theta}_{g}$ includes parameters of the limit-state function. The probability of failure, now a function of $\boldsymbol{\theta}_{f}$ and $\boldsymbol{\theta}_{g}$, is given by

$$
P_{f}\left(\boldsymbol{\theta}_{f}, \boldsymbol{\theta}_{g}\right)=\int_{g\left(\mathbf{x}, \boldsymbol{\theta}_{g}\right) \leq 0} f_{\mathbf{X}}\left(\mathbf{x}, \boldsymbol{\theta}_{f}\right) d \mathbf{x}
$$

For the sake of simplicity, we will consider the case where the two parameter vectors reduce to a scalar $\theta$, with $\theta$ representing either a distribution or a limitstate parameter. The probability of failure is then denoted $P_{f}(\theta)$. The discussion can be directly extended to address the general case of Equation (3).

If $\theta$ is a distribution parameter, the derivative of
$P_{f}(\theta)$ is given by

$\frac{d P_{f}(\theta)}{d \theta}=\int_{g(\mathbf{x}) \leq 0} \frac{\partial f_{\mathbf{X}}(\mathbf{x}, \theta)}{\partial \theta} d \mathbf{x}$

It is seen that the above is a (possibly high dimensional) domain integral and can be solved by application of standard Monte Carlo methods. This has been acknowledged by Wu (1994), who applied standard importance sampling for the solution of Equation (4). Other variance reduction methods can also be applied, such as subset simulation (Song et al. 2009). However, it should be noted that these approaches cannot be applied in the $\mathbf{U}$-space. This can be understood by considering that in the $\mathbf{U}$-space, distribution parameters enter the definition of the transformed limit-state function $G($.$) , whereas the \operatorname{PDF} \varphi_{n}($.$) is parameter$ free.

A different approach was introduced by Melchers \& Ahammed (2004) for the estimation of distribution parameter sensitivities. The authors constructed a linear response surface using Monte Carlo samples and applied the FORM sensitivity results to this surrogate model. It is noted here that this approach can also be applied to evaluate sensitivities to limit-state parameters, since the latter are also provided as byproducts of FORM.

In the case where $\theta$ is a limit-state parameter, the derivative of $P_{f}(\theta)$ is given by the following surface integral (Breitung 1994)

$$
\frac{d P_{f}(\theta)}{d \theta}=-\int_{g(\mathbf{x}, \theta)=0} f_{\mathbf{X}}(\mathbf{x}) \frac{1}{\left\|\nabla_{\mathbf{x}} g(\mathbf{x}, \theta)\right\|} \frac{\partial g(\mathbf{x}, \theta)}{\partial \theta} d s(\mathbf{x})
$$

where $d s(\mathbf{x})$ denotes surface integration over the surface $g(\mathbf{x}, \theta)=0$. Classical Monte Carlo methods cannot be applied for the estimation of surface integrals. However, the integral in Equation (5) can be estimated by simulation methods that monitor the boundary of the limit-state function. Such methods are the directional sampling (Bjerager 1988) and line sampling (Hohenbichler \& Rackwitz 1988, Koutsourelakis et al. 2004). Both approaches require the solution of a line search problem for each sample to determine the intersection of the sampling direction or line with the limit-state surface. Application of these methods to reliability sensitivity will be discussed in the following section.

Alternative methods for solving the problem of Equation (5) have been proposed in Royset \& Polak (2004) and in Jensen et al. (2009). The procedure introduced by Royset \& Polak (2004) requires solving the limit-state equation $g(\mathbf{x}, \theta)=0$ for one component of $\mathbf{x}$. Estimation of the reliability sensitivity is achieved by conditional importance sampling in terms 
of the remaining random variables. The method presented in Jensen et al. (2009) involves a linear approximation of the limit-state function in terms of the parameter vector and an approximation of the probability of failure in terms of the limit-state function. The approximations are constructed perturbing the limitstate parameters at the samples close to the failure surface and performing additional limit-state function evaluations.

In this paper, we introduce a novel approach for approximate reliability sensitivity analysis with standard Monte Carlo methods. This approach is based on an approximation of the surface integral of Equation (5) in terms of a domain integral. The method is discussed in detail in a subsequent section and its performance is investigated with a numerical example.

\section{RELIABILITY SENSITIVITY WITH EXISTING VARIANCE REDUCTION METHODS}

\subsection{Directional sampling}

Directional sampling (Bjerager 1988) is a simulation method for estimation of the probability of failure in the $\mathbf{U}$-space. Application of the method is limited to reliability problems with star-shaped limit-states. As already noted, in the $\mathbf{U}$-space the dependence of either distribution or limit-state parameters enters the definition of the transformed limit-state function $G($.$) . Us-$ ing a polar coordinate transformation $\mathbf{U}=R \mathbf{A}$, where $\mathbf{A}$ is a unit direction vector uniformly distributed on the $n$-dimensional hypersphere and $R$ is the radius in the direction A, the integral of Equation (2) is expressed as

$$
\begin{aligned}
P_{f}(\theta) & =\int_{D(\mathbf{a})} \int_{r(\mathbf{a}, \theta)}^{\infty} f_{R}(s) d s f_{\mathbf{A}}(\mathbf{a}) d \mathbf{a} \\
& =\int_{D(\mathbf{a})}\left[1-F_{R}(r(\mathbf{a}, \theta))\right] f_{\mathbf{A}}(\mathbf{a}) d \mathbf{a}
\end{aligned}
$$

wherein the dependency on the parameter $\theta$ has been included. $f_{\mathbf{A}}$ denotes the uniform distribution on the $n$-dimensional unit hypersphere, $f_{R}$ and $F_{R}$ are the PDF and CDF of the $\chi$ distribution with $n$ degrees of freedom, $D(\mathbf{a})$ is the unit hypersphere and $r(\mathbf{a}, \theta)$ is the distance from the origin to the boundary of the failure domain in the direction a, i.e. $G(r(\mathbf{a}, \theta) \mathbf{a})=0$, $r(\mathbf{a}, \theta) \in \mathbb{R}_{+}$(see Fig. 1). Equation (6) can be viewed as an expectation with respect to the PDF $f_{\mathbf{A}}$. Hence, $P_{f}(\theta)$ can be estimated by generating $n_{s}$ independent samples $\left\{\mathbf{a}_{k}, k=1, \ldots, n_{s}\right\}$ of $\mathbf{A}$ and taking the sample mean of $\left[1-F_{R}(r(\mathbf{a}, \theta))\right]$, i.e.

$P_{f}(\theta) \approx \frac{1}{n_{s}} \sum_{k=1}^{n_{s}}\left[1-F_{R}\left(r\left(\mathbf{a}_{k}, \theta\right)\right)\right]$

For each sample $\mathbf{a}_{k}$, the root of $G\left(r\left(\mathbf{a}_{k}, \theta\right) \mathbf{a}_{k}\right)=0$ can be found by application of any line-search method.

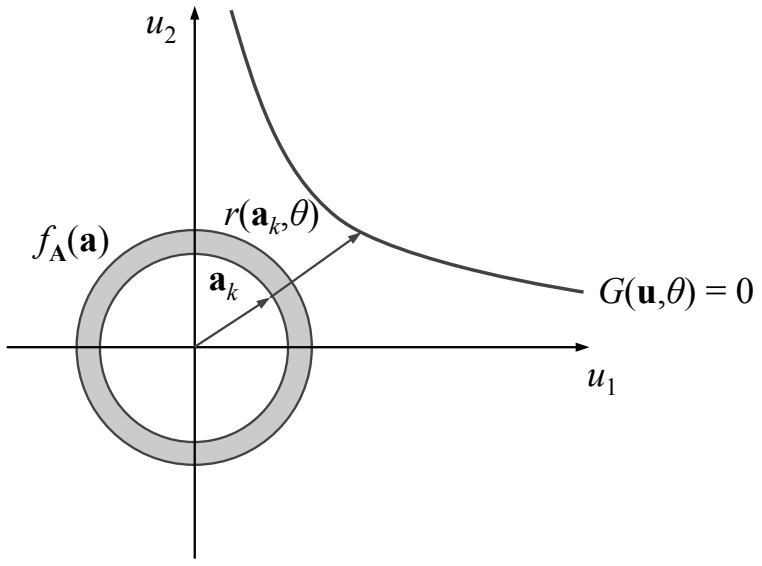

Figure 1: Graphical representation of directional sampling in a 2D standard normal space.

Taking the derivative of Equation (6) with respect to $\theta$, we have

$$
\frac{d P_{f}(\theta)}{d \theta}=\int_{D(\mathbf{a})}\left[-f_{R}(r(\mathbf{a}, \theta)) \frac{\partial r(\mathbf{a}, \theta)}{\partial \theta}\right] f_{\mathbf{A}}(\mathbf{a}) d \mathbf{a}
$$

The intersection point of the direction a with the limitstate surface is $\mathbf{u}(\mathbf{a}, \theta)=r(\mathbf{a}, \theta) \mathbf{a}$. It follows that

$$
\frac{\partial \mathbf{u}(\mathbf{a}, \theta)}{\partial \theta}=\frac{\partial r(\mathbf{a}, \theta)}{\partial \theta} \mathbf{a}
$$

Linearization of $G(\mathbf{u}(\mathbf{a}, \theta))=0$ yields

$$
\frac{\partial G(\mathbf{u}(\mathbf{a}, \theta))}{\partial \theta}+\nabla_{\mathbf{u}} G(\mathbf{u}(\mathbf{a}, \theta))^{T} \frac{\partial \mathbf{u}(\mathbf{a}, \theta)}{\partial \theta}=0
$$

Combining Equations (9) and (10), we get

$$
\frac{\partial r(\mathbf{a}, \theta)}{\partial \theta}=-\frac{1}{\nabla_{\mathbf{u}} G(\mathbf{u}(\mathbf{a}, \theta))^{T} \mathbf{a}} \frac{\partial G(\mathbf{u}(\mathbf{a}, \theta))}{\partial \theta}
$$

Substituting Equation (11) to Equation (8), we get

$$
\begin{aligned}
& \frac{d P_{f}(\theta)}{d \theta} \\
& \quad=\int_{D(\mathbf{a})}\left[\frac{f_{R}(r(\mathbf{a}, \theta))}{\nabla_{\mathbf{u}} G(\mathbf{u}(\mathbf{a}, \theta))^{T} \mathbf{a}} \frac{\partial G(\mathbf{u}(\mathbf{a}, \theta))}{\partial \theta}\right] f_{\mathbf{A}}(\mathbf{a}) d \mathbf{a}
\end{aligned}
$$

Taking sample mean of the term in brackets from the samples $\left\{\mathbf{a}_{k}, k=1, \ldots, n_{s}\right\}$, we finally obtain an estimate of the sensitivity of the probability of failure

$$
\frac{d P_{f}(\theta)}{d \theta} \approx \frac{1}{n_{s}} \sum_{k=1}^{n_{s}}\left[\frac{f_{R}\left(r\left(\mathbf{a}_{k}, \theta\right)\right)}{\nabla_{\mathbf{u}} G\left(\mathbf{u}\left(\mathbf{a}_{k}, \theta\right)\right)^{T} \mathbf{a}_{k}} \frac{\partial G\left(\mathbf{u}\left(\mathbf{a}_{k}, \theta\right)\right)}{\partial \theta}\right]
$$

The term in the denominator is the cosine between the sampling direction and the gradient of the limit-state function at the intersection point. Hence, evaluation of the gradient of the limit-state function is required at each sampling direction. 


\subsection{Line sampling}

Line sampling or axis parallel importance sampling was introduced in Hohenbichler \& Rackwitz (1988) for obtaining a correction factor to FORM/SORM estimates of the probability of failure by sampling parallel to the direction pointing to the most probable failure point. The method was further applied in Koutsourelakis et al. (2004) replacing the initial FORM/SORM run with a coarse Monte Carlo simulation to determine the sampling direction. The method solves the reliability problem in the $\mathbf{U}$-space. Therein, the sampling space is rotated and reduced by one dimension, i.e. $\mathbf{V}=\mathbf{R} \mathbf{U}, \mathbf{V}=\left[\mathbf{V}_{1}^{T}, V_{n}\right]^{T}$, where $\mathbf{V}_{1}$ contains the $(n-1)$-dimensional subspace, $V_{n}$ is the coordinate parallel to a direction a pointing to the limitstate surface and $\mathbf{R}$ is a suitable rotation matrix, whose $n$th row is the unit direction a. The probability integral is expressed as

$$
\begin{aligned}
P_{f}(\theta) & =\int_{D\left(\mathbf{v}_{1}\right)} \int_{b\left(\mathbf{v}_{1}, \theta\right)}^{\infty} \varphi\left(v_{n}\right) d v_{n} \varphi_{n-1}\left(\mathbf{v}_{1}\right) d \mathbf{v}_{1} \\
& =\int_{D\left(\mathbf{v}_{1}\right)} \Phi\left(-b\left(\mathbf{v}_{1}, \theta\right)\right) \varphi_{n-1}\left(\mathbf{v}_{1}\right) d \mathbf{v}_{1}
\end{aligned}
$$

where $\varphi_{n-1}$ is the $(n-1)$-variate standard normal PDF on the hyperplane $v_{n}=0, \varphi$ and $\Phi$ are the standard normal PDF and CDF, $D\left(\mathbf{v}_{1}\right)=\mathbb{R}^{n-1}$ and $b\left(\mathbf{v}_{1}, \theta\right)$ is the distance to the failure surface in a direction orthogonal to the hyperplane $v_{n}=0$ at $\mathbf{v}_{1}$, i.e. the solution of $G\left(\mathbf{R}^{T}\left[\mathbf{v}_{1}^{T}, b\left(\mathbf{v}_{1}, \theta\right)\right]^{T}\right)=0$ (see Fig. 2). $P_{f}(\theta)$ can be estimated by generating $n_{s}$ independent samples $\left\{\mathbf{v}_{1 k}, k=1, \ldots, n_{s}\right\}$ of $\mathbf{V}_{1}$ and applying

$P_{f}(\theta) \approx \frac{1}{n_{s}} \sum_{k=1}^{n_{s}} \Phi\left(-b\left(\mathbf{v}_{1 k}, \theta\right)\right)$

where for each sample $\mathbf{v}_{1 k}$, the root of $G\left(\mathbf{R}^{T}\left[\mathbf{v}_{1}^{T}, b\left(\mathbf{v}_{1}, \theta\right)\right]^{T}\right)=0$ is found by application of a line-search method.

The derivative of Equation (14) reads

$$
\frac{d P_{f}(\theta)}{d \theta}=\int_{D\left(\mathbf{v}_{1}\right)}\left[-\varphi\left(b\left(\mathbf{v}_{1}, \theta\right)\right) \frac{\partial b\left(\mathbf{v}_{1}, \theta\right)}{\partial \theta}\right] \varphi_{n-1}\left(\mathbf{v}_{1}\right) d \mathbf{v}_{1}
$$

The intersection point of the line parallel to $\mathbf{a}$ at $\mathbf{v}_{1}$ is $\mathbf{u}\left(\mathbf{v}_{1}, \theta\right)=\mathbf{R}^{T}\left[\mathbf{v}_{1}^{T}, b\left(\mathbf{v}_{1}, \theta\right)\right]^{T}$. Taking derivatives and accounting for the fact that the $n$th column of $\mathbf{R}^{T}$ is the vector $\mathbf{a}$, we get

$$
\frac{\partial \mathbf{u}\left(\mathbf{v}_{1}, \theta\right)}{\partial \theta}=\frac{\partial b\left(\mathbf{v}_{1}, \theta\right)}{\partial \theta} \mathbf{a}
$$

Linearization of $G\left(\mathbf{u}\left(\mathbf{v}_{1}, \theta\right)\right)=0$ yields

$\frac{\partial G\left(\mathbf{u}\left(\mathbf{v}_{1}, \theta\right)\right)}{\partial \theta}+\nabla_{\mathbf{u}} G\left(\mathbf{u}\left(\mathbf{v}_{1}, \theta\right)\right)^{T} \frac{\partial \mathbf{u}\left(\mathbf{v}_{1}, \theta\right)}{\partial \theta}=0$

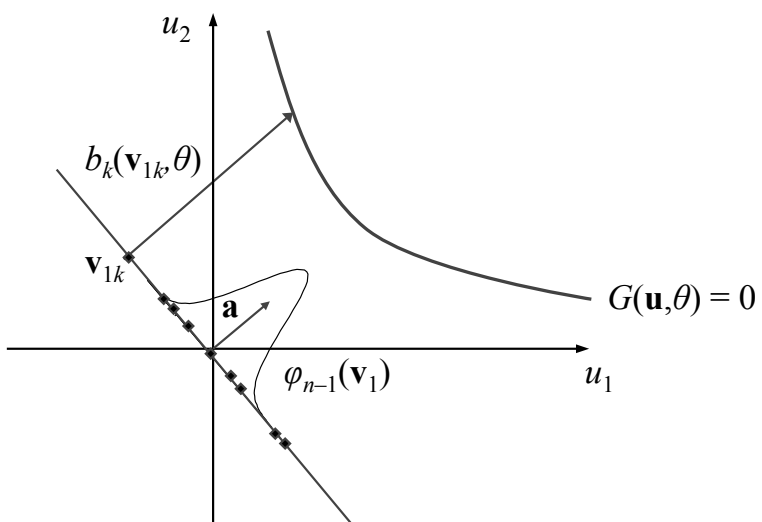

Figure 2: Graphical representation of line sampling in a 2D standard normal space.

Combining Equations (17) and (18), we get

$\frac{\partial b\left(\mathbf{v}_{1}, \theta\right)}{\partial \theta}=-\frac{1}{\nabla_{\mathbf{u}} G\left(\mathbf{u}\left(\mathbf{v}_{1}, \theta\right)\right)^{T} \mathbf{a}} \frac{\partial G\left(\mathbf{u}\left(\mathbf{v}_{1}, \theta\right)\right)}{\partial \theta}$

Substituting Equation (19) to Equation (16), we get

$$
\begin{aligned}
& \frac{d P_{f}(\theta)}{d \theta} \\
& =\int_{D\left(\mathbf{v}_{1}\right)}\left[\frac{\varphi\left(b\left(\mathbf{v}_{1}, \theta\right)\right)}{\nabla_{\mathbf{u}} G\left(\mathbf{u}\left(\mathbf{v}_{1}, \theta\right)\right)^{T} \mathbf{a}} \frac{\partial G\left(\mathbf{u}\left(\mathbf{v}_{1}, \theta\right)\right)}{\partial \theta}\right] \varphi_{n-1}\left(\mathbf{v}_{1}\right) d \mathbf{v}_{1}
\end{aligned}
$$

Taking sample mean of the term in brackets from the samples $\left\{\mathbf{v}_{1 k}, k=1, \ldots, n_{s}\right\}$, we obtain an estimate of the sensitivity of the probability of failure

$$
\frac{d P_{f}(\theta)}{d \theta} \approx \frac{1}{n_{s}} \sum_{k=1}^{n_{s}}\left[\frac{\varphi\left(b\left(\mathbf{v}_{1 k}, \theta\right)\right)}{\nabla_{\mathbf{u}} G\left(\mathbf{u}\left(\mathbf{v}_{1 k}, \theta\right)\right)^{T} \mathbf{a}} \frac{\partial G\left(\mathbf{u}\left(\mathbf{v}_{1 k}, \theta\right)\right)}{\partial \theta}\right]
$$

The term in the denominator is the cosine between the direction $\mathbf{a}$ and the gradient of the limit-state function at the intersection point. Therefore, evaluation of the gradient of the limit-state function is again required at each sampling line.

\section{APPROXIMATE RELIABILITY SENSITIVITY WITH MONTE CARLO}

\subsection{Approximation of the surface integral}

In this section we derive an approximation of the surface integral of Equation (5) in terms of a domain integral. The reliability problem of Equation (1) can be written as follows

$P_{f}(\theta)=\int_{D(\mathbf{x})} I(\mathbf{x}) f_{\mathbf{X}}(\mathbf{x}) d \mathbf{x}$

where $D(\mathbf{x})=\mathbb{R}^{n}$ and $I(\mathbf{x})$ is the indicator function of the failure domain. Standard Monte Carlo estimates $P_{f}(\theta)$ by generating $n_{s}$ samples $\left\{\mathbf{x}_{k}, k=1, \ldots, n_{s}\right\}$ of $\mathbf{X}$ and taking the sample mean of $I(\mathbf{X})$, i.e.

$P_{f}(\theta) \approx \frac{1}{n_{s}} \sum_{k=1}^{n_{s}} I\left(\mathbf{x}_{k}\right)$ 
The indicator function can be expressed by the following limit [e.g. see (Spanier \& Oldham 1987)]

$I(\mathbf{x})=\lim _{\sigma \rightarrow 0} \Phi\left(-\frac{g(\mathbf{x}, \theta)}{\sigma}\right)$

where $\Phi$ is the standard normal CDF. Choosing $\sigma$ small enough, we can approximate $I(\mathbf{x})$ by the following expression

$I(\mathbf{x}) \approx \Phi\left(-\frac{g(\mathbf{x}, \theta)}{\sigma}\right)$

This is illustrated in Figure 3 where $\Phi\left(-\frac{g}{\sigma}\right)$ is plotted for different values of $\sigma$. Inserting Equation (25) into Equation (22), we obtain an approximation of the probability of failure denoted by $\tilde{P}_{f}(\theta, \sigma)$ and expressed as follows

$\tilde{P}_{f}(\theta, \sigma)=\int_{D(\mathbf{x})} \Phi\left(-\frac{g(\mathbf{x}, \theta)}{\sigma}\right) f_{\mathbf{X}}(\mathbf{x}) d \mathbf{x}$

Taking the derivative of the above with respect to $\theta$, we get

$$
\frac{\partial \tilde{P}_{f}(\theta, \sigma)}{\partial \theta}=-\int_{D(\mathbf{x})} \frac{1}{\sigma} \varphi\left(\frac{g(\mathbf{x}, \theta)}{\sigma}\right) \frac{\partial g(\mathbf{x}, \theta)}{\partial \theta} f_{\mathbf{X}}(\mathbf{x}) d \mathbf{x}
$$

Equation (27) is a domain integral and hence can be estimated using the Monte Carlo samples $\left\{\mathbf{x}_{k}, k=\right.$ $\left.1, \ldots, n_{s}\right\}$, as follows

$$
\frac{\partial \tilde{P}_{f}(\theta, \sigma)}{\partial \theta} \approx \frac{1}{n_{s}} \sum_{k=1}^{n_{s}}\left[-\frac{1}{\sigma} \varphi\left(\frac{g\left(\mathbf{x}_{k}, \theta\right)}{\sigma}\right) \frac{\partial g\left(\mathbf{x}_{k}, \theta\right)}{\partial \theta}\right]
$$

\subsection{Convergence of approximation as $\sigma \rightarrow 0$}

It is clear that the approximation of the probability of failure $\tilde{P}_{f}(\theta, \sigma)$ converges to $P_{f}(\theta)$ as $\sigma \rightarrow 0$. We now show that under some regularity conditions, the approximation in Equation (27) converges to the true surface integral in Equation (5) as $\sigma \rightarrow 0$. To show this, we change variables in the integral of Equation (27) by applying the mapping $\mathbf{x} \rightarrow(\mathbf{y}, h)$, where $\mathbf{y}$ is the coordinate system on the surface $g(\mathbf{x}, \theta)=0$ and $h$ is the distance normal to the surface. We then truncate the integration domain at a distance $d$ to the limit-state surface, leading to the following approximation

$$
\frac{\partial \tilde{P}_{f}(\theta, \sigma)}{\partial \theta} \approx-\int_{g(\mathbf{y}, \theta)=0} \int_{-d}^{d} \frac{1}{\sigma} \varphi\left(\frac{g(\mathbf{y}+h \mathbf{n}(\mathbf{y}), \theta)}{\sigma}\right) \times
$$

$\frac{\partial g(\mathbf{y}+h \mathbf{n}(\mathbf{y}), \theta)}{\partial \theta} J^{-1}(\mathbf{y}+h \mathbf{n}(\mathbf{y})) d h f_{\mathbf{X}}(\mathbf{y}) d s(\mathbf{y})$

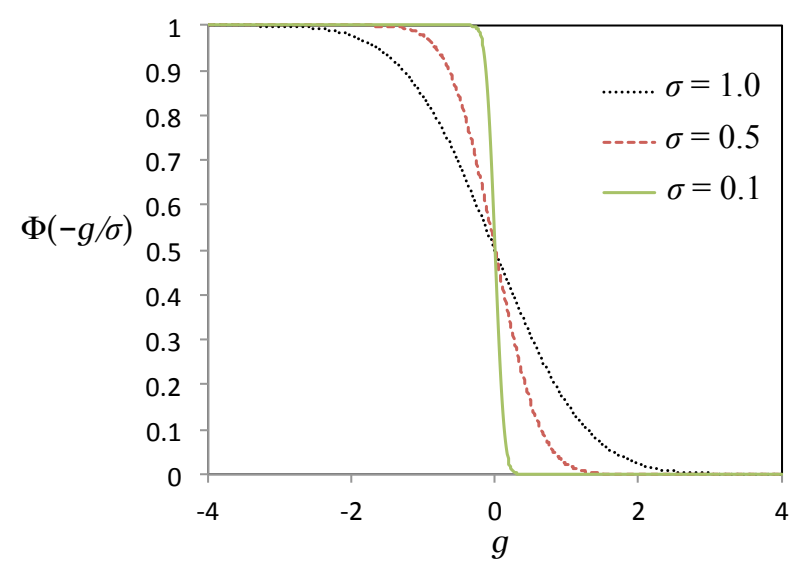

Figure 3: Approximation of the indicator function for different values of $\sigma$.

where $\mathbf{n}(\mathbf{y})=\left\|\nabla_{\mathbf{y}} g(\mathbf{y}, \theta)\right\|^{-1} \nabla_{\mathbf{y}} g(\mathbf{y}, \theta)$ and $J^{-1}($.$) is$ the Jacobian of the inverse mapping $(\mathbf{y}, h) \rightarrow \mathbf{x}$. Since $\mathbf{n}(\mathbf{y})$ is orthogonal to the limit-state surface at $\mathbf{y}$ one has always that $J^{-1}(\mathbf{y}, 0)=1$. Therefore, we have

$\frac{\partial \tilde{P}_{f}(\theta, \sigma)}{\partial \theta}$

$\approx-\int_{g(\mathbf{y}, \theta)=0}\left[\int_{-d}^{d} \frac{1}{\sigma} \varphi\left(\frac{g(\mathbf{y}+h \mathbf{n}(\mathbf{y}), \theta)}{\sigma}\right) d h\right] \times$

$\frac{\partial g(\mathbf{y}, \theta)}{\partial \theta} f_{\mathbf{X}}(\mathbf{y}) d s(\mathbf{y})$

The integral over $h$ can be approximated making a Taylor expansion of $g$ at $\mathbf{y}$, which gives

$$
\begin{aligned}
& \int_{-d}^{d} \frac{1}{\sigma} \varphi\left(\frac{g(\mathbf{y}+h \mathbf{n}(\mathbf{y}), \theta)}{\sigma}\right) d h \\
& \quad \approx \int_{-d}^{d} \frac{1}{\sigma} \varphi\left(\frac{g(\mathbf{y}, \theta)+h\left\|\nabla_{\mathbf{y}} g(\mathbf{y}, \theta)\right\|}{\sigma}\right) d h
\end{aligned}
$$

The above follows from the first order multivariate Taylor expansion $g(\mathbf{y}+h \mathbf{n}(\mathbf{y}))-g(\mathbf{y}, \theta) \approx$ $h \mathbf{n}(\mathbf{y})^{T} \nabla_{\mathbf{y}} g(\mathbf{y}, \theta)=h\left\|\nabla_{\mathbf{y}} g(\mathbf{y}, \theta)\right\|$. Since $\mathbf{y}$ runs on the limit-state surface, one has $g(\mathbf{y}, \theta)=0$ in Equation (31). The resulting integral can be written as

$$
\begin{aligned}
& \int_{-d}^{d} \frac{1}{\sigma} \varphi\left(\frac{h\left\|\nabla_{\mathbf{y}} g(\mathbf{y}, \theta)\right\|}{\sigma}\right) d h \\
& =\frac{1}{\left\|\nabla_{\mathbf{y}} g(\mathbf{y}, \theta)\right\|}\left[\Phi\left(\frac{d\left\|\nabla_{\mathbf{y}} g(\mathbf{y}, \theta)\right\|}{\sigma}\right)-\right. \\
& \left.\Phi\left(-\frac{d\left\|\nabla_{\mathbf{y}} g(\mathbf{y}, \theta)\right\|}{\sigma}\right)\right]
\end{aligned}
$$

The term in brackets in the above converges towards unity as $\sigma \rightarrow 0$. Inserting this into Equation (30) shows that $\frac{\partial \tilde{P}_{f}(\theta, \sigma)}{\partial \theta}$ converges to $\frac{d P_{f}(\theta)}{d \theta}$ as $\sigma \rightarrow 0$. 


\subsection{Discussion on the choice of $\sigma$}

The quality of the approximation of Equation (27) depends on the choice of the parameter $\sigma$. As shown in Section 4.2, the bias of the approximation decreases with decreasing value of $\sigma$. However, a very small $\sigma$ would lead to a high coefficient of variation in the Monte Carlo estimate of Equation (28). This is due to the fact that for small $\sigma$, most of the samples values away from the limit-state surface will be zero. Hence, an optimal choice of $\sigma$ should achieve a compromise between the bias and the coefficient of variation of the corresponding Monte Carlo estimate. Here, we illustrate this with the help of a simple numerical example.

Consider the limit-state function $g(u, \theta)=-u+\beta$, where $u$ is the outcome of a standard normal random variable and $\beta$ is a deterministic parameter. It is noted that any linear function of normal random variables can be reduced to a similar form. The probability of failure equals $\Phi(-\beta)$ and the sensitivity with respect to $\beta$ is $-\varphi(\beta)$. Let us assume that the probability of failure is estimated with crude Monte Carlo, with a target coefficient of variation $5 \%$. Knowing the true value of the probability of failure, we can estimate the required number of samples $n_{s} \approx 400 / \Phi(-\beta)$. Therefore, for a given value of $\beta$, the integral of Equation (27) as well as the coefficient of variation of the estimate of Equation (28) can be evaluated.

Figure 4 plots the relative bias, i.e. the absolute difference between the approximation and the true value of the sensitivity divided by the absolute value of the latter, the coefficient of variation of the sensitivity estimate and the Euclidean norm of the two, for $\beta=3.5$ and $\beta=4.0$ and different values of the parameter $\sigma$. The plots show that the minimum value of the norm of the two error measures, which corresponds to the optimal choice of $\sigma$, would be acceptable for most problems, which supports the feasibility of the approach. Moreover, it is shown that the optimal value of $\sigma$ leads to slightly larger coefficient of variation than the one of the probability estimate. It is noted, however, that for this example the derivative of the limit-state function in terms of the parameter equals unity, which is not the general case. In general, the behavior of this derivative at the vicinity of the limit-state surface is shown to greatly affect the optimal choice of $\sigma$.

In practical situations, the optimal value of $\sigma$ cannot be computed, since the true value of the probability sensitivity is not known and hence the relative bias of the approximation cannot be estimated. Therefore, it is suggested to vary the value of $\sigma$ and choose the minimum $\sigma$ that leads to a coefficient of variation smaller than a target value. It is noted that the same samples of the limit-state function and its derivative with respect to the parameter can be used for estimation of the coefficient of variation for different values of $\sigma$. Therefore, the additional computational cost for this adaptive procedure is negligible.
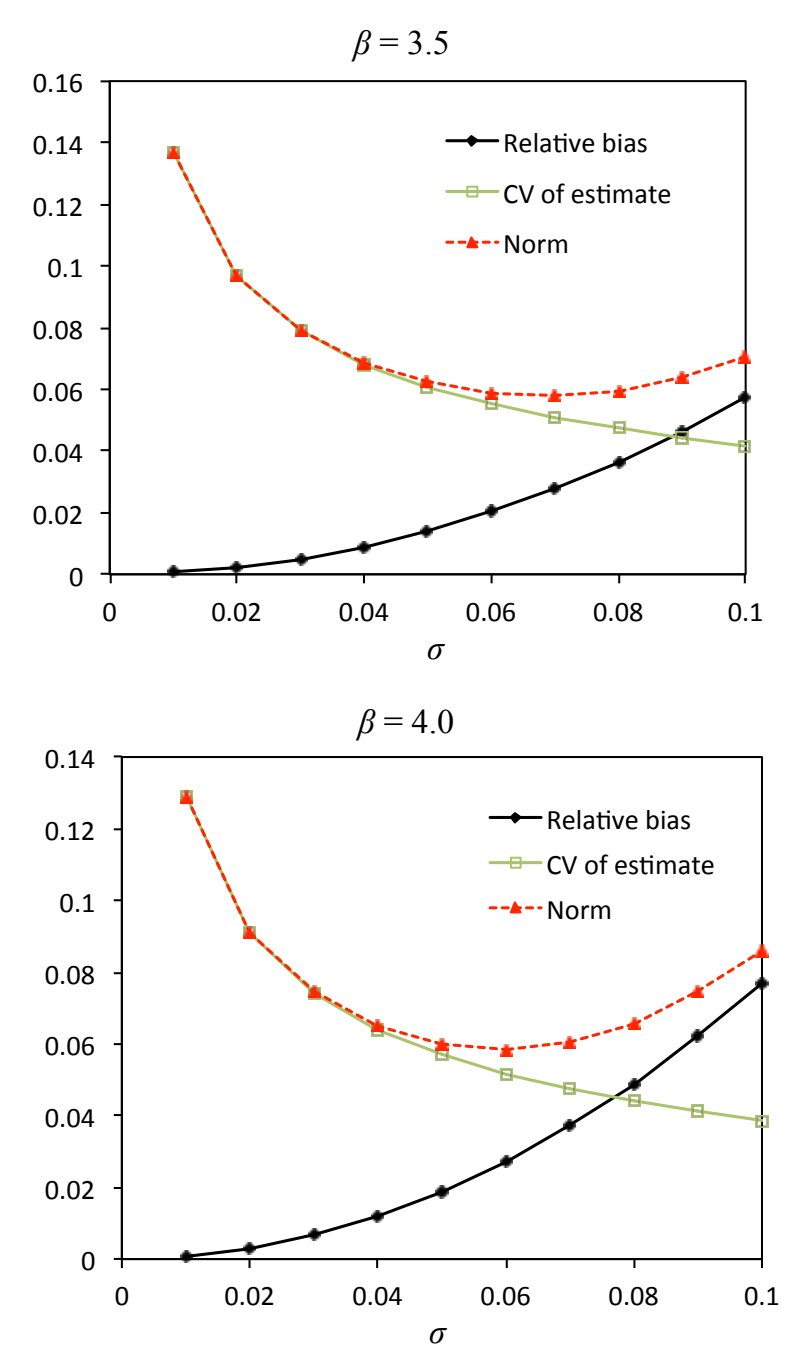

Figure 4: Relative bias and coefficient of variation of estimate of parameter sensitivity; Linear limit-state in terms of normal random variable and in terms of limit-state parameter.

\section{EXAMPLE}

The performance of the Monte Carlo methods discussed here is demonstrated with the help of a numerical example, presented in Yang \& Gu (2004) in the context of reliability-based optimization. The example consists of a cantilever beam subjected to biaxial bending (Fig. 5). The loads and material properties of the beam are modeled by independent normal random variables, as shown in Table 1. Two limit-state functions are considered, representing different failure modes of the beam. The first one represents yielding at the fixed end of the cantilever:

$g_{1}(Y, X, y, w, t)=y-\left(\frac{600}{w t^{2}} Y+\frac{600}{w^{2} t} X\right)$

The second limit-state function restricts the maximum allowed displacements at the tip of the beam:

$$
\begin{aligned}
g_{2}\left(Y, X, E, w, t, D_{0}\right)= & D_{0} \\
& -\frac{4 L^{3}}{E w t} \sqrt{\left(\frac{Y}{t^{2}}\right)^{2}+\left(\frac{X}{w^{2}}\right)^{2}}
\end{aligned}
$$



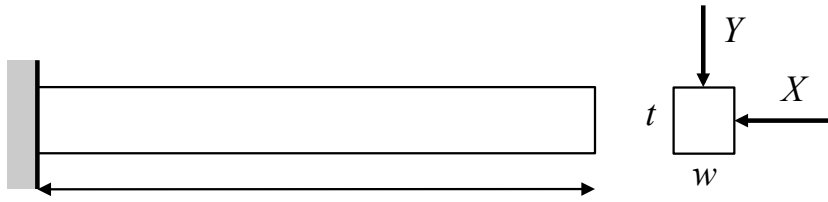

$L=100^{\prime \prime}$

Figure 5: Cantilever beam under biaxial bending

The parameters of the limit-state functions are the width $w$ and height $t$ of the cross-section of the beam and the allowable tip displacement $D_{0}$. The parameter sensitivities of the probability of failure are estimated at $\left(w, t, D_{0}\right)=(2.4,3.9,2.5)$ [in], which corresponds to the reliability-based design for an allowable probability of failure of $3 \times 10^{-3}$.

We present results obtained by directional sampling, line sampling as well as estimates of the proposed approximation obtained by an importance sampling method. The latter involves an initial Monte Carlo run that estimates the point closer to the limitstate surface with minimum distance to the origin of the $\mathbf{U}$-space and a subsequent importance sampling run with importance sampling density centered at this point. It is noted that the efficiency of this approach depends on the importance sampling density estimated by the initial Monte Carlo run. The parameter $\sigma$ of the approximation is evaluated following the adaptive procedure described in Section 4.3 with a target coefficient of variation of $5 \%$ for the sensitivity estimates. In the line sampling, the sampling direction is also estimated by an initial Monte Carlo run. Moreover, the results are compared with the probability sensitivities obtained by FORM.

Table 2 shows the results for the limit-state function $g_{1}$. The function $g_{1}$ is linear in the normal random variables and therefore the results obtained by FORM will be exact. The results obtained by directional and line sampling show excellent agreement with the FORM results, with the line sampling being the most efficient of the two. It is noted, however, that both methods require the evaluation of the gradient of the limit-state function in terms of the random variables at each sample. The estimates of the proposed approximation with importance sampling are very close to the exact sensitivity results. In Tables 2 and 3, the number of samples of the initial Monte Carlo run are indicated for the line sampling and importance sampling methods.

In Table 3, the reliability sensitivities obtained for the limit-state function $g_{2}$ are shown. This function is nonlinear in all three random variables, therefore the FORM results are approximate. The results obtained by line sampling are considered closer to the exact sensitivities due to their small coefficient of variation. Directional sampling produces estimates very close to the ones by line sampling, although with a significantly larger number of samples required to achieve a small coefficient of variation. The estimates based on the proposed approximation with importance sam-
Table 1: Uncertain parameters of the cantilever beam.

\begin{tabular}{llll}
\hline Parameter & Distribution & Mean & CV \\
\hline Load $X[\mathrm{lb}]$ & Normal & 500 & $20 \%$ \\
Load $Y[\mathrm{lb}]$ & Normal & 1000 & $10 \%$ \\
Yield strength $y$ [psi] & Normal & 40000 & $5 \%$ \\
Young's modulus $E$ [psi] & Normal & $29 \times 10^{6}$ & $5 \%$ \\
\hline
\end{tabular}

pling are again close to the exact results, further indicating that the proposed approximation can be used for reliability sensitivity estimation to acceptable accuracy.

\section{CONCLUSION}

This paper discussed Monte Carlo methods for the estimation of reliability sensitivities to limit-state parameters. The application of two existing variance reduction techniques to reliability sensitivity estimation, namely directional and line sampling, was considered and it was pointed out that both methods require the evaluation of the gradient of the limit-state function in terms of the random variables at each sample. Standard Monte Carlo cannot be applied to the estimation of sensitivities to limit-state parameters, since these consist of surface integrals over the limitstate surface. An approximation of the surface integral through a domain integral was introduced, which allows application of standard Monte Carlo as well as importance sampling techniques for estimation. The applicability of the approximation in conjunction with an importance sampling strategy was demonstrated through a numerical application.

Finally, it should be noted that estimation of the proposed approximation requires the derivative of the limit-state function in terms of the parameters at each sample. In problems involving limit-state functions expressed in terms of numerical models this task can involve significant additional computational cost. However, an optimization of the method aiming at reducing the number of derivative evaluations can be attempted, since the contribution of most samples away from the limit-state surface is insignificant. This task is left to future research.

\section{REFERENCES}

Bjerager, P. (1988). Probability integration by directional simulation. J. Eng. Mech.-ASCE 114, 1285-1302.

Breitung, K. (1994). Asymptotic approximations of probability integrals, Volume 1592 of Lect. Notes Math. Berlin: Springer.

Der Kiureghian, A. \& P. L. Liu (1986). Structural reliability under incomplete probability information. J. Eng. Mech.ASCE 112(1), 85-104.

Ditlevsen, O. \& H. O. Madsen (1996). Structural Reliability Methods. Chichester: Wiley.

Hohenbichler, M. \& R. Rackwitz (1981). Non-normal dependent vectors in structural safety. J. Eng. Mech. Div.-ASCE 107(6), 1227-1238.

Hohenbichler, M. \& R. Rackwitz (1988). Improvements of second-order reliability estimates by importance sampling. 
Table 2: Parameter sensitivities for limit-state function $g_{1}$.

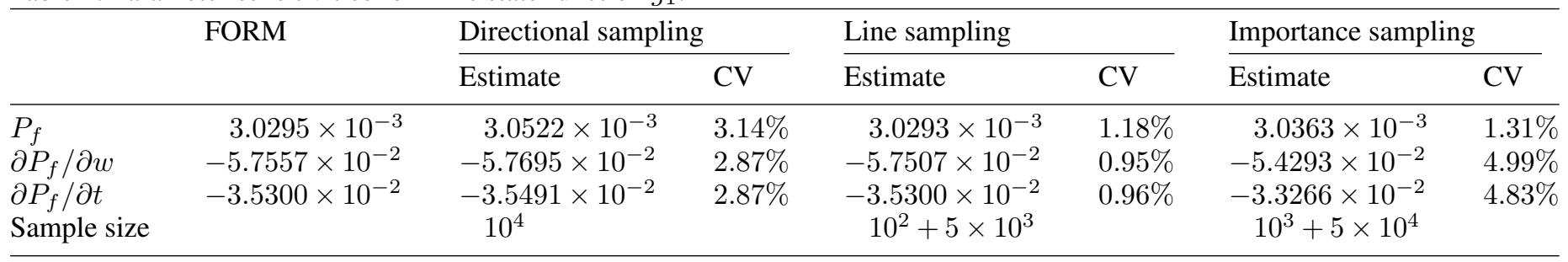

Table 3: Parameter sensitivities for limit-state function $g_{2}$.

\begin{tabular}{|c|c|c|c|c|c|c|c|}
\hline & \multirow[t]{2}{*}{ FORM } & \multicolumn{2}{|c|}{ Directional sampling } & \multicolumn{2}{|l|}{ Line sampling } & \multicolumn{2}{|c|}{ Importance sampling } \\
\hline & & Estimate & $\mathrm{CV}$ & Estimate & $\mathrm{CV}$ & Estimate & $\mathrm{CV}$ \\
\hline$P_{f}$ & $2.4115 \times 10^{-4}$ & $2.5031 \times 10^{-4}$ & $3.65 \%$ & $2.5200 \times 10^{-4}$ & $1.71 \%$ & $2.5240 \times 10^{-4}$ & $1.12 \%$ \\
\hline$\partial P_{f} / \partial w$ & $-8.4861 \times 10^{-3}$ & $-8.7554 \times 10^{-3}$ & $3.34 \%$ & $-8.8434 \times 10^{-3}$ & $1.41 \%$ & $-9.3640 \times 10^{-3}$ & $4.33 \%$ \\
\hline$\partial P_{f} / \partial t$ & $-2.8081 \times 10^{-3}$ & $-2.9264 \times 10^{-3}$ & $3.34 \%$ & $-2.9446 \times 10^{-3}$ & $1.50 \%$ & $-3.1117 \times 10^{-3}$ & $4.17 \%$ \\
\hline$\partial P_{f} / \partial D_{0}$ & $-3.1318 \times 10^{-3}$ & $-3.2426 \times 10^{-3}$ & $3.34 \%$ & $-3.2708 \times 10^{-3}$ & $1.44 \%$ & $-3.4633 \times 10^{-3}$ & $4.27 \%$ \\
\hline Sample size & & $2 \times 10^{4}$ & & $10^{2}+10^{4}$ & & $10^{3}+10^{5}$ & \\
\hline
\end{tabular}

J. Eng. Mech.-ASCE 114, 2195-2199.

Jensen, H. A., M. A. Valdebenito, G. I. Schuëller, \& D. S. Kusanovic (2009). Reliability-based optimization of stochastic systems using line search. Comput. Method. Appl. Mech. Eng. 198, 3915-3924.

Koutsourelakis, P. S., H. J. Pradlwarter, \& G. I. Schuëller (2004). Reliability of structures in high dimensions, part I: algorithms and applications. Probabilist. Eng. Mech. 19, 409417.

Lemaire, M. (2009). Structural Reliability. Hoboken, NJ: Wiley. Melchers, R. E. \& M. Ahammed (2004). A fast approximate method for parameter sensitivity estimation in monte carlo structural reliability. Comput. Struct. 82, 55-61.

Rackwitz, R. (2001). Reliability analysis - a review and some perspectives. Struct. Saf. 23, 365-395.

Royset, J. O. \& E. Polak (2004). Reliability-based optimal design using sample average approximations. Probabilist. Eng. Mech. 19, 331-343.

Song, S., Z. Lu, \& H. Qiao (2009). Subset simulation for structural reliability sensitivity analysis. Reliab. Eng. Syst. Safe. 94, 658-665.

Spanier, J. \& K. B. Oldham (1987). An Atlas of Functions. Washington, DC: Hemisphere.

Valdebenito, M. A., H. J. Pradlwarter, \& G. I. Schuëller (2010). The role of the design point for calculating failure probabilities in view of dimensionality and structural nonlinearities. Struct. Saf. 32, 101-111.

Wu, Y.-T. (1994). Comutational methods for efficient structural reliability and reliability sensitivity analysis. AIAA J. 32(8), 1717-1723.

Yang, R. J. \& L. Gu (2004). Experience with approximate reliability-based optimization methods. Struct. Multidisc. Optim. 26, 152-159. 